\title{
Special Issue in Honor of Professor Chi-Wang Shu
}

\author{
Sigal Gottlieb ${ }^{1} \cdot$ Johnny Guzmán ${ }^{2} \cdot$ Fengyan $\mathrm{Li}^{3}$ • \\ Jennifer K. Ryan ${ }^{4}$
}

Published online: 12 October 2017

(C) Springer Science+Business Media, LLC 2017



With great joy and enthusiasm, we are proud to present this special issue of the Journal of Scientific Computing, in honor of Professor Chi-Wang Shu on the occasion of his sixtieth birthday. The theme of this issue is High Order Methods, which is a perfect fit with the distin-

\footnotetext{
Fengyan Li

lif@rpi.edu

Sigal Gottlieb

sgottlieb@umassd.edu

Johnny Guzmán

Johnny_Guzman@brown.edu

Jennifer K. Ryan

Jennifer.Ryan@uea.ac.uk

1 Department of Mathematics, University of Massachusetts at Dartmouth, North Dartmouth, MA 02747, USA

2 Division of Applied Mathematics, Brown University, Providence, RI 02912, USA

3 Department of Mathematical Sciences, Rensselaer Polytechnic Institute, Troy, NY 12180, USA

4 School of Mathematics, University of East Anglia, Norwich NR4 7TJ, UK
} 
guished contributions of Chi-Wang Shu in developing numerical methods, especially of high order accuracy. His numerous contributions to the field include TVD temporal discretizations, ENO and WENO finite difference/volume schemes, discontinuous Galerkin methods and spectral methods, and encompass both theoretical analysis and applications.

Chi-Wang Shu received his B.S. degree from the University of Science and Technology of China (1982) and his Ph.D. degree from the University of California at Los Angeles (1986), both in Mathematics. After a year as a postdoc fellow at the Institute for Mathematics and Its Applications (IMA), he joined the Division of Applied Mathematics at Brown University, and currently holds the Theodore B. Stowell University Professorship. Chi-Wang's work in the past three and half decades has made great impact on Scientific Computing and Numerical Analysis. This has been recognized and acknowledged in numerous ways, including the NASA Public Service Group Achievement Award (1992) for his pioneering work in Computational Fluid Dynamics, the first Feng Kang Prize of Scientific Computing from the Chinese Academy of Sciences (1995), the SIAM/ACM Prize in Computational Science and Engineering (2007), being elected a member of the inaugural class of Fellows of the Society for Industrial and Applied Mathematics (SIAM 2009) and the American Mathematical Society (AMS 2012). Since 2004 Chi-Wang has been listed as an ISI Highly Cited Author in Mathematics by the ISI Web of Knowledge.

Chi-Wang has also made tremendous contributions to the scientific community by mentoring and supporting junior researchers, including his Ph.D. students, postdocs, visiting scholars, collaborators and colleagues, and even participants of his numerous summer school lectures, with quite many taking up academic appointments. Many who have interacted with Chi-Wang will resonate with the personal messages delivered at the conference by some of his students: "Words cannot express my gratitude for everything you have taught me, and for all your support throughout the years", "You have been a mentor, a role model and an inspiration, and I am fortunate to be your student", "Thank you for enlightening my career and my life", "You are the wisest and nicest man that I have ever met", "I could not ask for a better academic family".

Besides his friends, students and postdocs, the contributors to this special issue include the distinguished invited speakers of the international conference, Frontiers in Applied and Computational Mathematics, held during January 4-6, 2017, Providence RI. The stellar list of attendees includes 20 world-renowned invited speakers representing a diverse range of topics represented by them, as well as the 124 participants from 11 countries at various career stages. The number and diversity of the participants are a reflection of the high esteem in which ChiWang is held in the international scientific computing community. More importantly, they also show a warm and supportive community Chi-Wang has created. 